https://doi.org/10.15407/ujpe63.6.488

A.M. PAVLYUK

Bogolyubov Institute for Theoretical Physics, Nat. Acad. of Sci. of Ukraine

(14b, Metrolohichna Str., Kyiv03143, Ukraine; e-mail: pavlyuk@bitp.kiev.ua)

\title{
GENERALIZED EQUIDISTANT CHEBYSHEV POLYNOMIALS AND ALEXANDER KNOT INVARIANTS ${ }^{1}$
}

\begin{abstract}
We introduce the generalized equidistant Chebyshev polynomials $T^{(k, h)}$ of kind $k$ of hyperkind $h$, where $k, h$ are positive integers. They are obtained by a generalization of standard and monic Chebyshev polynomials of the first and second kinds. This generalization is fulfilled in two directions. The horizontal generalization is made by introducing hyperkind $h$ and expanding it to infinity. The vertical generalization proposes expanding kind $k$ to infinity with the help of the method of equidistant coefficients. Some connections of these polynomials with the Alexander knot and link polynomial invariants are investigated.

Keywords: Chebyshev polynomials, generalization, kind, hyperkind, equidistant coefficients, recurrence relation, knots and links, Alexander polynomial invariants.
\end{abstract}

\section{Introduction}

Knots and links appear in many areas of physics, particulary in electrodynamics, optics, liquid crystals, hydrodynamics [1-4]. The main characteristics of knots and links follow from the axioms of skein relation and normalization, which lead to polynomial invariants [5]. Each knot and link is described by some definite polynomial. The most known polynomial invariants are Alexander [6], Jones [7] and HOMFLY [8] ones.

The Chebyshev polynomials appear in different branches of mathematics, particulary in approximation theory, knot theory, combinatorics, differential equations, number theory [9]. As important mathematical instruments, they widely penetrate into different branches of physics. We exploit the Chebyshev polynomials and their generalizations for the description of knots and links [10-12]. To our mind, they have to play important role even as in the searching of new polynomial invariants.

In the present paper, we introduce the generalized equidistant Chebyshev polynomials $T^{(k, h)}$ of

(C) A.M. PAVLYUK, 2018 kind $k$ of hyperkind $h$, where $k, h$ are positive integers. At the beginning, we unify the standard Chebyshev polynomials of the first kind and monic Chebyshev polynomials of the first kind with the help of one formula using the newly introduced notion of hyperkind $h$ ( $h=1$ refers to all standard Chebyshev polynomials; $h=2$ - to all monic Chebyshev polynomials). After that, a generalization means declaring $h$ to be any positive integer and leads to generalized Chebyshev polynomials of the first kind. Analogously, one obtains generalized Chebyshev polynomials of the second kind. Thus, we fulfil the so-called horizontal generalization. The final step is making the vertical generalization, by using the method of equidistant coefficients. As a result, we expand the meanings of kind $k$ to all positive integers and obtain the formula for generalized equidistant Chebyshev polynomials of kind $k$ of hyperkind $h$.

1 This paper was presented at the 3rd Walter Thirring International School on Fundamentals of Astroparticle and Quantum Physics (17-23 September, 2017, BITP, Kyiv, Ukraine).

ISSN 2071-0194. Ukr. J. Phys. 2018. Vol. 63, No. 6 


\section{Standard Chebyshev Polynomials}

The standard Chebyshev polynomials of the first kind $T_{n}^{(1)}(x)$ are introduced as

$T_{n}^{(1)}(x)=\cos (n \theta), \quad x=\cos \theta$,

where $n$ is a nonnegative integer. In our paper, the Chebyshev polynomials are characterized by two numbers - $(k, h)$. Here, the letter $k$ stands for "kind". In addition, we introduce a new notion of "hyperkind", for which we use the upper index $h$ (in brackets); $h=1$ refers to all standard Chebyshev polynomials. Thus, for all polynomials defined by (1), $(k, h)=(1,1)$. In the case of monic Chebyshev polynomials considered in the next section, $h=2$. The relation

$\cos (n+1) \theta+\cos (n-1) \theta=2 \cos \theta \cos n \theta$

yields the recurrence relation for the standard Chebyshev polynomials of the first kind:

$T_{n+1}^{(1)}(x)=2 x T_{n}^{(1)}(x)-T_{n-1}^{(1)}(x), T_{0}^{(1)}=1, T_{1}^{(1)}=x$,

which can be considered as an alternative definition of $T_{n}^{(1)}(x)$. Some examples of $T_{n}^{(1)}(x)$ obtained from (3) are as follows:

$T_{0}^{(1)}=1, T_{1}^{(1)}=x, T_{2}^{(1)}=2 x^{2}-1$,

$T_{3}^{(1)}=4 x^{3}-3 x, T_{4}^{(1)}=8 x^{4}-8 x^{2}+1$,

$T_{5}^{(1)}=16 x^{5}-20 x^{3}+5 x$.

The standard Chebyshev polynomials of the second kind $V_{n}^{(1)}(x)$ are defined in the following way $(k=2$, $h=1)$ :

$V_{n}^{(1)}(x)=\frac{\sin (n+1) \theta}{\sin \theta}, \quad x=\cos \theta$.

The relation

$\sin (n+2) \theta+\sin n \theta=2 \cos \theta \sin (n+1) \theta$

implies that definition (5) can be written in terms of the recurrence relation similar to $(3)$ :

$V_{n+1}^{(1)}(x)=2 x V_{n}^{(1)}(x)-V_{n-1}^{(1)}(x), V_{0}^{(1)}=1, V_{1}^{(1)}=2 x$.
Some first standard Chebyshev polynomials of the second kind are:

$V_{0}^{(1)}=1, V_{1}^{(1)}=2 x, V_{2}^{(1)}=4 x^{2}-1$,

$V_{3}^{(1)}=8 x^{3}-4 x, V_{4}^{(1)}=16 x^{4}-12 x^{2}+1$,

$V_{5}^{(1)}=32 x^{5}-32 x^{3}+6 x$.

From the formula

$2 \cos (n \theta)=\left(\frac{\sin (n+1) \theta}{\sin \theta}-\frac{\sin (n-1) \theta}{\sin \theta}\right)$,

one finds that definition (1) of $T_{n}^{(1)}(x)$ can be rewritten in the form

$T_{n}^{(1)}(x)=\frac{1}{2} \frac{\sin (n+1) \theta}{\sin \theta}-\frac{1}{2} \frac{\sin (n-1) \theta}{\sin \theta}, \quad x=\cos \theta$.

As follows from (9), the Chebyshev polynomials $T_{n}^{(1)}(x)$ and $V_{n}^{(1)}(x)$ are connected by the formula

$2 T_{n}^{(1)}(x)=V_{n}^{(1)}(x)-V_{n-2}^{(1)}(x), \quad n \geq 0$.

Here, we mean that $V_{-1}^{(1)}=0, V_{-2}^{(1)}=-1$, which follows from (7)

\section{Monic Chebyshev Polynomials}

The monic Chebyshev polynomials of the first kind $T_{n}^{(2)}(x),(k=1, h=2)$, are defined by

$T_{n}^{(2)}(x)=2 \cos (n \theta), \quad x=2 \cos \theta$.

The monic normalization means that all Chebyshev polynomials have the unit coefficients at the highest degree $x^{n}$.

The recurrence relation

$T_{n+1}^{(2)}(x)=x T_{n}^{(2)}(x)-T_{n-1}^{(2)}(x), T_{0}^{(2)}=2, T_{1}^{(2)}=x$,

can be considered as an alternative definition of $T_{n}^{(2)}(x)$. Some first cases of $T_{n}^{(2)}(x)$ are:

$T_{0}^{(2)}=2, T_{1}^{(2)}=x, T_{2}^{(2)}=x^{2}-2$,

$T_{3}^{(2)}=x^{3}-3 x, T_{4}^{(2)}=x^{4}-4 x^{2}+2$,

$T_{5}^{(2)}=x^{5}-5 x^{3}+5 x$.

The monic Chebyshev polynomials of the second kind $V_{n}^{(2)}(x)$ are defined as $(k=2, h=2)$

$V_{n}^{(2)}(x)=\frac{\sin (n+1) \theta}{\sin \theta}, \quad x=2 \cos \theta$ 
or, in terms of the recurrence relation,

$V_{n+1}^{(2)}(x)=x V_{n}^{(2)}(x)-V_{n-1}^{(2)}(x), V_{0}^{(2)}=1, V_{1}^{(2)}=x$.

The first monic Chebyshev polynomials of the second kind are:

$V_{0}^{(2)}=1, V_{1}^{(2)}=x, V_{2}^{(2)}=x^{2}-1$,

$V_{3}^{(2)}=x^{3}-2 x, V_{4}^{(2)}=x^{4}-3 x^{2}+1$,

$V_{5}^{(2)}=x^{5}-4 x^{3}+3 x$.

Definition (12) of $T_{n}^{(2)}(x)$ can be written in the form

$T_{n}^{(2)}(x)=\frac{\sin (n+1) \theta}{\sin \theta}-\frac{\sin (n-1) \theta}{\sin \theta}, x=2 \cos \theta$.

The Chebyshev polynomials $T_{n}^{(2)}$ and $V_{n}^{(2)}$ are connected by the formula

$T_{n}^{(2)}=V_{n}^{(2)}-V_{n-2}^{(2)}, \quad n \geq 0$,

where $V_{-1}^{(2)}=0, V_{-2}^{(2)}=-1$, being calculated from (16).

\section{Horizontal Generalization of Chebyshev Polynomials: Expanding Hyperkind $h$ to Infinity}

The main idea for the horizontal generalization of Chebyshev polynomials consists of two steps. The first step is a unification of standard Chebyshev polynomials and monic Chebyshev polynomials of the same kinds. At the beginning, we unify $(k=1, h=1)$ and ( $k=1, h=2$ ) Chebyshev polynomials with the help of one formula; another formula unifies $(k=2$, $h=1)$ and $(k=2, h=2)$ Chebyshev polynomials. These formulas automatically lead to the second step of the horizontal generalization, by expanding the meanings of hyperkind $h$ to infinity.

\subsection{Generalized Chebyshev polynomials of the first kind}

Thus, comparing (1) and (12), we write down the unified Chebyshev polynomials of the first kind $T_{n}^{(h)}(x)$ by the formula:

$T_{n}^{(h)}(x)=h \cos (n \theta), \quad x=h \cos \theta$,

for $h=1$ and $h=2$. From this formula, one obtains the generalized Chebyshev polynomials of the first kind, by expanding $h$ to infinity, i.e., $h$ being any positive integer: $h=1,2,3,4, \ldots$.

Relation (2) written in the form

$h \cos (n+1) \theta+h \cos (n-1) \theta=\frac{2}{h} h \cos \theta h \cos n \theta$

yields the recurrence relation for the generalized Chebyshev polynomials of the first kind

$T_{n+1}^{(h)}(x)=\frac{2}{h} x T_{n}^{(h)}(x)-T_{n-1}^{(h)}(x)$,

$T_{0}^{(h)}=h, T_{1}^{(h)}=x$,

which can be taken for the definition of $T_{n}^{(h)}(x)$.

Some examples of $T_{n}^{(h)}(x)$ obtained from (21) are:

$T_{0}^{(h)}=h, T_{1}^{(h)}=x, T_{2}^{(h)}=\frac{2}{h} x^{2}-h$,

$T_{3}^{(h)}=\frac{4}{h^{2}} x^{3}-3 x, T_{4}^{(h)}=\frac{8}{h^{3}} x^{4}-\frac{8}{h} x^{2}+h$,

$T_{5}^{(h)}=\frac{16}{h^{4}} x^{5}-\frac{20}{h^{2}} x^{3}+5 x$.

From (20), (21), and (22), one has formulas for the standard Chebyshev polynomials of the first kind, if $h=1$, and for the monic Chebyshev polynomials of the first kind in the case $h=2$.

As an example, let us write (generalized) Chebyshev polynomials of the first kind of the third hyperkind. For $(k=1, h=3)$, one has

$T_{n}^{(3)}(x)=3 \cos (n \theta), 3 \cos \theta=x$,

$T_{n+1}^{(3)}=\frac{2}{3} x T_{n}^{(3)}-T_{n-1}^{(3)}, T_{0}^{(3)}=3, T_{1}^{(3)}=x$,

$T_{0}^{(3)}=3, T_{1}^{(3)}=x, T_{2}^{(3)}=\frac{2}{3} x^{2}-3$,

$T_{3}^{(3)}=\frac{4}{9} x^{3}-3 x, T_{4}^{(3)}=\frac{8}{27} x^{4}-\frac{8}{3} x^{2}+3$,

$T_{5}^{(3)}=\frac{16}{81} x^{5}-\frac{20}{9} x^{3}+5 x$.

As follows from (9), definition (20) of $T_{n}^{(h)}(x)$ can be also written as

$T_{n}^{(h)}(x)=\frac{h}{2} \frac{\sin (n+1) \theta}{\sin \theta}-\frac{h}{2} \frac{\sin (n-1) \theta}{\sin \theta}, x=h \cos \theta$.

\subsection{Generalized Chebyshev polynomials of the second kind}

Comparing (5) and (15), we obtain firstly unified Chebyshev polynomials of the second kind (for $h=1$ and $h=2$ ). Second, these formulas are used to introduce

ISSN 2071-0194. Ukr. J. Phys. 2018. Vol. 63, No. 6 
the generalized ones by declaring the hyperkind $h$ to be any positive integer.

Thus, the generalized Chebyshev polynomials of the second kind $V_{n}^{(h)}(x)$ are defined as

$V_{n}^{(h)}(x)=\frac{\sin (n+1) \theta}{\sin \theta}, \quad x=h \cos \theta$,

with $h$ to be a positive integer. Relation (6) yields definition (27) in terms of the recurrence relation

$V_{n+1}^{(h)}=\frac{2}{h} x V_{n}^{(h)}-V_{n-1}^{(h)}, V_{0}^{(h)}=1, V_{1}^{(h)}=\frac{2}{h} x$.

In view of (28), some first generalized Chebyshev polynomials of the second kind are as follows:

$$
\begin{aligned}
V_{0}^{(h)} & =1, V_{1}^{(h)}=\frac{2}{h} x, V_{2}^{(h)}=\frac{4}{h^{2}} x^{2}-1, \\
V_{3}^{(h)} & =\frac{8}{h^{3}} x^{3}-\frac{4}{h} x, V_{4}^{(h)}=\frac{16}{h^{4}} x^{4}-\frac{12}{h^{2}} x^{2}+1, \\
V_{5}^{(h)} & =\frac{32}{h^{5}} x^{5}-\frac{32}{h^{3}} x^{3}+\frac{6}{h} x .
\end{aligned}
$$

By substituting $h=1$ into (29), one has standard Chebyshev polynomials of the second kind; if $h=2-$ monic ones.

From formula (9), one finds that Chebyshev polynomials $T_{n}^{(h)}(x)$ and $V_{n}^{(h)}(x)$ are connected by the formula

$2 T_{n}^{(h)}(x)=h\left(V_{n}^{(h)}(x)-V_{n-2}^{(h)}(x)\right), \quad n \geq 0$,

where $V_{-1}^{(h)}=0, V_{-2}^{(h)}=-1$ from $(28)$.

For example, (generalized) Chebyshev polynomials of the second kind of the third hyperkind, $(k=2$, $h=3$ ), are:

$$
\begin{aligned}
& V_{n}^{(3)}(x)=\frac{\sin (n+1) \theta}{\sin \theta}, \quad 3 \cos \theta=x, \\
& V_{n+1}^{(3)}=\frac{2}{3} x V_{n}^{(3)}-V_{n-1}^{(3)}, V_{0}^{(3)}=1, V_{1}^{(3)}=\frac{2}{3} x, \\
& V_{0}^{(3)}=1, V_{1}^{(3)}=\frac{2}{3} x, V_{2}^{(3)}=\frac{4}{9} x^{2}-1, \\
& V_{3}^{(3)}=\frac{8}{27} x^{3}-\frac{4}{3} x, V_{4}^{(3)}=\frac{16}{81} x^{4}-\frac{12}{9} x^{2}+1, \\
& V_{5}^{3}=\frac{32}{243} x^{5}-\frac{32}{27} x^{3}+2 x .
\end{aligned}
$$

\section{Vertical Generalization of Chebyshev Polynomials: Expanding Kind $k$ to Infinity}

The next direction of a generalization of Chebyshev polynomials is the vertical generalization. This means

ISSN 2071-0194. Ukr. J. Phys. 2018. Vol. 63, No. 6 expanding the meaning of kind $k$ to infinity. The main idea for the vertical generalization is the method of equidistant coefficients.

We now introduce the following notation for generalized Chebyshev polynomials: $T_{n}^{(k, h)}(x)$. They are connected with the abovementioned polynomials in such way: $T_{n}^{(1,1)}(x)=T_{n}^{(1)}(x), T_{n}^{(1,2)}(x)=T_{n}^{(2)}(x)$, $T_{n}^{(1, h)}(x)=T_{n}^{(h)}(x), T_{n}^{(2,1)}(x)=V_{n}^{(1)}(x), T_{n}^{(2,2)}(x)=$ $=V_{n}^{(2)}(x), T_{n}^{(2, h)}(x)=V_{n}^{(h)}(x)$. Now, we are going to generalize standard (and monic) Chebyshev polynomials by expanding the meaning of kind $k$.

\subsection{Equidistant standard Chebyshev polynomials}

The polynomials introduced here can be also called generalized Chebyshev polynomials of the first hyperkind or equidistant Chebyshev polynomials of the first hyperkind.

As an example, let us introduce the standard Chebyshev polynomials of the third kind $T_{n}^{(3,1)}(x)$, i.e., $(k==3, h=1)$. Comparing the introductions of $T_{n}^{(1,1)}(x)$ by (3) and $T_{n}^{(2,1)}(x)$ by (7) (i.e., $T_{n}^{(1)}(x)$ and $V_{n}^{(1)}(x)$ ), one obtains, as a result of the natural generalization, the following definition:

$T_{n+1}^{(3,1)}(x)=2 x T_{n}^{(3,1)}(x)-T_{n-1}^{(3,1)}(x)$,

$T_{0}^{(3,1)}=1, T_{1}^{(3,1)}=3 x$.

Some examples of $T_{n}^{(3,1)}(x)$ are:

$T_{0}^{(3,1)}=1, T_{1}^{(3,1)}=3 x, T_{2}^{(3,1)}=6 x^{2}-1$,

$T_{3}^{(3,1)}=12 x^{3}-5 x, T_{4}^{(3,1)}=24 x^{4}-16 x^{2}+1$,

$T_{5}^{(3,1)}=48 x^{5}-44 x^{3}+7 x$.

By using (4), (8), and (35), it is easy to verify that

$T_{n}^{(3,1)}-T_{n}^{(2,1)}=T_{n}^{(2,1)}-T_{n}^{(1,1)}$,

$n=1,2,3,4,5$,

which demonstrates the property of equidistancy of the coefficients.

By analogy, the definition of standard Chebyshev polynomials of arbitrary $\operatorname{kind} T_{n}^{(k, 1)}(x)$ is:

$T_{n+1}^{(k, 1)}(x)=2 x T_{n}^{(k, 1)}(x)-T_{n-1}^{(k, 1)}(x)$,

$T_{0}^{(k, 1)}=1, \quad T_{1}^{(k, 1)}=k x$. 
Some first examples of $T_{n}^{(k, 1)}(x)$ are:

$T_{0}^{(k, 1)}=1, T_{1}^{(k, 1)}=k x$,

$T_{2}^{(k, 1)}=2 k x^{2}-1$,

$T_{3}^{(k, 1)}=4 k x^{3}-(k+2) x$,

$T_{4}^{(k, 1)}=8 k x^{4}-4(k+1) x^{2}+1$,

$T_{5}^{(k, 1)}=16 k x^{5}-4(3 k+2) x^{3}+(k+4) x$.

The equidistancy of the coefficients in this case is expressed by the fact that the difference

$T_{n}^{(k+1,1)}-T_{n}^{(k, 1)}$

is independent of $k$ for any $n$ (due to the linearity of $T_{n}^{(k, 1)}$ in $\left.k\right)$.

Putting $k=1$ in (37), one has (4), $k=2$ gives (8), and $k=3-(35)$.

Taking (10) and (5) in the form

$$
\begin{aligned}
& T_{n}^{(1,1)}(x)=\frac{1}{2} \frac{\sin (n+1) \theta}{\sin \theta}-\frac{1}{2} \frac{\sin (n-1) \theta}{\sin \theta}, \\
& T_{n}^{(2,1)}(x)=1 \frac{\sin (n+1) \theta}{\sin \theta}+0 \frac{\sin (n-1) \theta}{\sin \theta}, \\
& x=\cos \theta,
\end{aligned}
$$

and using the property of equidistant coefficiens, one easily finds the formula for equidistant standard Chebyshev polynomials of the third kind (of the first hyperkind):

$$
\begin{aligned}
& T_{n}^{(3,1)}(x)=\frac{3}{2} \frac{\sin (n+1) \theta}{\sin \theta}+\frac{1}{2} \frac{\sin (n-1) \theta}{\sin \theta}, \\
& x=\cos \theta,
\end{aligned}
$$

and of arbitrary kind of the first hyperkind:

$$
\begin{aligned}
& T_{n}^{(k, 1)}(x)=\frac{k}{2} \frac{\sin (n+1) \theta}{\sin \theta}+\frac{(k-2)}{2} \frac{\sin (n-1) \theta}{\sin \theta}, \\
& x=\cos \theta
\end{aligned}
$$

It is easy to verify, by using (6), that (39) and (40) satisfy (34) and (36), correspondingly.

At last, we prove (40). Indeed, the general formula has two equidistant coefficients $\alpha^{(k, 1)}$ and $\beta^{(k, 1)}$ and looks as

$T_{n}^{(k, 1)}(x)=\alpha^{(k, 1)} \frac{\sin (n+1) \theta}{\sin \theta}+\beta^{(k, 1)} \frac{\sin (n-1) \theta}{\sin \theta}$.

Introducing the difference of neighboring equidistant coefficients

$\delta_{1}^{(h)}=\alpha^{(k+1, h)}-\alpha^{(k, h)}, \delta_{2}^{(h)}=\beta^{(k+1, h)}-\beta^{(k, h)}$, which are idependent of $k$, we have

$$
\begin{aligned}
& \alpha^{(k, 1)}=\alpha^{(1,1)}+\delta_{1}^{(1)}(k-1)=\alpha^{(1,1)}+\left(\alpha^{(2,1)}-\alpha^{(1,1)}\right) \times \\
& \times(k-1)=\frac{1}{2}+\left(1-\frac{1}{2}\right)(k-1)=\frac{k}{2}, \\
& \beta^{(k, 1)}=\beta^{(1,1)}+\delta_{2}^{(1)}(k-1)=\beta^{(1,1)}+\left(\beta^{(2,1)}-\beta^{(1,1)}\right) \times \\
& \times(k-1)=-\frac{1}{2}+\left(0-\left(-\frac{1}{2}\right)\right)(k-1)=\frac{(k-2)}{2} .
\end{aligned}
$$

\subsection{Equidistant monic Chebyshev polynomials}

They can be also called generalized Chebyshev polynomials of the second hyperkind or equidistant Chebyshev polynomials of the second hyperkind.

To introduce the monic Chebyshev polynomials of the third kind $T_{n}^{(3,2)}(x)$, where $(k=3, h=2)$, one must compare the introductions of $T_{n}^{(1,2)}(x)$ by (13) and $T_{n}^{(2,2)}(x)$ by (16):

$T_{n+1}^{(3,2)}(x)=x T_{n}^{(3,2)}(x)-T_{n-1}^{(3,2)}(x)$,

$T_{0}^{(3,2)}=0, T_{1}^{(3,2)}=x$.

Below, we give some examples of $T_{n}^{(3,2)}(x)$ :

$T_{0}^{(3,2)}=0, T_{1}^{(3,2)}=x, T_{2}^{(3,2)}=x^{2}$,

$T_{3}^{(3,2)}=x^{3}-x, T_{4}^{(3,2)}=x^{4}-2 x^{2}$,

$T_{5}^{(3,2)}=x^{5}-3 x^{3}+x$.

Let us define monic Chebyshev polynomials of arbitrary kind $T_{n}^{(k, 2)}(x)$ :

$T_{n+1}^{(k, 2)}(x)=x T_{n}^{(k, 2)}(x)-T_{n-1}^{(k, 2)}(x)$,

$T_{0}^{(k, 2)}=3-k, T_{1}^{(k, 2)}=x$.

The first examples of $T_{n}^{(k, 2)}(x)$ are as follows:

$T_{0}^{(k, 2)}=3-k, T_{1}^{(k, 2)}=x$,

$T_{2}^{(k, 2)}=x^{2}+(k-3), T_{3}^{(k, 2)}=x^{3}+(k-4) x$,

$T_{4}^{(k, 2)}=x^{4}+(k-5) x^{2}-(k-3)$,

$T_{5}^{(k, 2)}=x^{5}+(k-6) x^{3}-(2 k-7) x$.

Comparing (18) and (15) in the form

$T_{n}^{(1,2)}(x)=1 \frac{\sin (n+1) \theta}{\sin \theta}-1 \frac{\sin (n-1) \theta}{\sin \theta}$,

$T_{n}^{(2,2)}(x)=1 \frac{\sin (n+1) \theta}{\sin \theta}+0 \frac{\sin (n-1) \theta}{\sin \theta}$, $x=2 \cos \theta$,

ISSN 2071-0194. Ukr. J. Phys. 2018. Vol. 63, No. 6 
and using equidistant coefficiens, one obtains the formula for equidistant monic Chebyshev polynomials of arbitrary kind:

$T_{n}^{(k, 2)}(x)=\frac{\sin (n+1) \theta}{\sin \theta}+(k-2) \frac{\sin (n-1) \theta}{\sin \theta}$,

$x=2 \cos \theta$.

\subsection{Equidistant Chebyshev} polynomials of kind $k$ of hyperkind $h$

Taking (26) and (27) in the form

$$
\begin{aligned}
& T_{n}^{(1, h)}(x)=\frac{h}{2} \frac{\sin (n+1) \theta}{\sin \theta}-\frac{h}{2} \frac{\sin (n-1) \theta}{\sin \theta}, \\
& T_{n}^{(2, h)}(x)=1 \frac{\sin (n+1) \theta}{\sin \theta}+0 \frac{\sin (n-1) \theta}{\sin \theta}, \\
& x=h \cos \theta,
\end{aligned}
$$

and using the property of equidistant coefficiens, one easily finds the formula for equidistant standard Chebyshev polynomials of arbitrary kind:

$$
\begin{aligned}
& T_{n}^{(k, h)}(x)=\left((k-1)-(k-2) \frac{h}{2}\right) \frac{\sin (n+1) \theta}{\sin \theta}+ \\
& +\left((k-2) \frac{h}{2}\right) \frac{\sin (n-1) \theta}{\sin \theta}, \quad x=h \cos \theta .
\end{aligned}
$$

Indeed, the general formula has two equidistant coefficients $\alpha^{(k, h)}$ and $\beta^{(k, h)}$ and looks as

$T_{n}^{(k, h)}(x)=\alpha^{(k, h)} \frac{\sin (n+1) \theta}{\sin \theta}+\beta^{(k, h)} \frac{\sin (n-1) \theta}{\sin \theta}$,

where

$$
\begin{aligned}
& \alpha^{(k, h)}=\alpha^{(1, h)}+\delta_{1}^{(h)}(k-1)= \\
& =\alpha^{(1, h)}+\left(\alpha^{(2, h)}-\alpha^{(1, h)}\right)(k-1)=(k-1)-(k-2) \frac{h}{2}, \\
& \beta^{(k, h)}=\beta^{(1, h)}+\delta_{2}^{(h)}(k-1)= \\
& \beta^{(1, h)}+\left(\beta^{(2, h)}-\beta^{(1, h)}\right)(k-1)=(k-2) \frac{h}{2} .
\end{aligned}
$$

From (21) and (49), we have

$$
\begin{aligned}
& T_{n+1}^{(k, h)}(x)=\frac{2}{h} x T_{n}^{(k, h)}(x)-T_{n-1}^{(k, h)}(x), \\
& T_{0}^{(k, h)}(x)=(k-1)-(k-2) h=A, \\
& T_{1}^{(k, h)}(x)=\left((k-1) \frac{2}{h}-(k-2)\right) x=B x,
\end{aligned}
$$

which can be the definition of $T_{n}^{(k, h)}(x)$.
At last, from (51), we have some first examples of $T_{n}^{(k, h)}(x)$ :

$$
\begin{aligned}
& T_{0}^{(k, h)}=A, T_{1}^{(k, h)}=B x, T_{2}^{(k, h)}=\frac{2}{h} B x^{2}-A, \\
& T_{3}^{(k, h)}=\frac{4}{h^{2}} B x^{3}-\left(\frac{2}{h} A+B\right) x, \\
& T_{4}^{(k, h)}=\frac{8}{h^{3}} B x^{4}-\left(\frac{4}{h^{2}} A+\frac{4}{h} B\right) x^{2}+A, \\
& T_{5}^{(k, h)}=\frac{16}{h^{4}} B x^{5}-\left(\frac{8}{h^{3}} A+\frac{12}{h^{2}} B\right) x^{3}+\left(\frac{4}{h} A+B\right) h .
\end{aligned}
$$

From (49), we obtain

$T_{n}^{(k, h)}=(k-1) T_{n}^{(2, h)}-(k-2) T_{n}^{(1, h)}$,

and

$T_{n}^{(k, h)}(x)=\left((k-1)-(k-2) \frac{h}{2}\right) T_{n}^{(2, h)}+$

$+\left((k-2) \frac{h}{2}\right) T_{n-2}^{(2, h)}, \quad x=h \cos \theta$.

\section{Chebyshev Polynomials and Alexander Knot Invariants}

The Alexander polynomials $\Delta(q)$ are defined by the Alexander skein relation for knots and links [6], (see notations in [13]):

$\Delta_{+}(q)-\Delta_{-}(q)=\left(q^{\frac{1}{2}}-q^{-\frac{1}{2}}\right) \Delta_{O}(q), \quad \Delta_{\text {unknot }}=1$.

The Alexander polynomial invariants for torus knots $T(n, l)$ are given by the formula $[14,15]$

$\Delta_{n, l}(q)=\frac{\left(q^{\frac{n l}{2}}-q^{-\frac{n l}{2}}\right)\left(q^{\frac{1}{2}}-q^{-\frac{1}{2}}\right)}{\left(q^{\frac{n}{2}}-q^{-\frac{n}{2}}\right)\left(q^{\frac{l}{2}}-q^{-\frac{l}{2}}\right)}$

where $n$ and $l$ are coprime positive integers. For $l=$ $=2$, formula (56) gives the expression for torus knots $T(n, 2)$ :

$\Delta_{n, 2}^{(K)}(q)=\frac{q^{\frac{n}{2}}+q^{-\frac{n}{2}}}{q^{\frac{1}{2}}+q^{-\frac{1}{2}}}, \quad n=2 m-1$,

where $m$ are positive integers: $m=1,2,3, \ldots$. Here, $(K)$ stands for a "knot" to be distinguished from a "link" $(L)$. Putting $q=e^{2 i \theta}$ in (57), we get

$\Delta_{n, 2}^{(K)}(q)=\frac{\cos n \theta}{\cos \theta}, \quad 2 \cos \theta=q^{\frac{1}{2}}+q^{-\frac{1}{2}}$. 
It easy to see that relations (20) and (58) yield

$\frac{T_{n}^{(1, h)}(x)}{x}=\frac{\cos n \theta}{\cos \theta}=\Delta_{n, 2}^{(K)}(q), \quad 2 \cos \theta=q^{\frac{1}{2}}+q^{-\frac{1}{2}}$

for odd $n$. This formula says firstly that the Alexander polynomials $\Delta_{n, 2}^{(K)}(q)$ are invariants (not depending on $h$ ) of all generalized Chebyshev polynomials of the first kind. Second, it gives the recipe for obtaining the corresponding Chebyshev polynomials (for odd $n$ ) from the Alexander polynomials $\Delta_{n, 2}^{(K)}(q)$ :

$T_{n}^{(1, h)}(x)=x \Delta_{n, 2}^{(K)}(q), \quad x=\frac{h}{2}\left(q^{\frac{1}{2}}+q^{-\frac{1}{2}}\right)$.

The corresponding formula for torus links $L(n, 2)$ (in the case of even $n$ ) [13] are:

$\Delta_{n, 2}^{(L)}(q)=\frac{q^{\frac{n}{2}}-q^{-\frac{n}{2}}}{q^{\frac{1}{2}}+q^{-\frac{1}{2}}}, \quad n=2 m$.

It can be shown that, for odd $n$,

$T_{n}^{(2, h)}(x)=\frac{2 x}{h} \frac{\Delta_{n+1,2}^{(L)}(q)}{\left(q^{\frac{1}{2}}-q^{-\frac{1}{2}}\right)}, \quad x=\frac{h}{2}\left(q^{\frac{1}{2}}+q^{-\frac{1}{2}}\right)$.

At last, we give some Alexander polynomials, which can be calculated from (57) and (61) and can be used for the direct verification of (60) and (62):

$$
\begin{aligned}
& \Delta_{1,2}^{(K)}(q)=1, \quad \Delta_{2,2}^{(L)}(q)=q^{\frac{1}{2}}-q^{-\frac{1}{2}}, \\
& \Delta_{3,2}^{(K)}(q)=q-1+q^{-1}, \\
& \Delta_{4,2}^{(L)}(q)=q^{\frac{3}{2}}-q^{\frac{1}{2}}+q^{-\frac{1}{2}}-q^{-\frac{3}{2}} \\
& \Delta_{5,2}^{(K)}(q)=q^{2}-q+1-q^{-1}+q^{-2} .
\end{aligned}
$$

\section{Concluding Remarks}

We have proposed a rather simple generalization of Chebyshev polynomials, which follows immediately from the attempt to unify standard and monic Chebyshev polynomials of both kinds with the help of one formula. Is is believed that these polynomials are connected with some knot and link polynomial invariants. Some connections of them with Alexander polynomial invariants are presented here. We believe that a deeper investigation in this direction will bring interesting results.

The present work was partially supported by the National Academy of Sciences of Ukraine (project No.0117U000238).
1. M.F. Atiyah. The Geometry and Physics of Knots (Cambridge Univ. Press, 1990).

2. L.H. Kauffman. Knots and Physics (World Scientific, 2001).

3. L.H. Kauffman (editor). The Interface of Knots and Physics. AMS Short Course Lecture Notes, a subseries of Proc. Symp. App. Math. 51 (AMS, 1996).

4. E. Radu, M.S. Volkov. Stationary ring solitons in field theory - knots and vortons. Phys. Rep. 468 (4), 101 (2008).

5. J.H. Conway. An enumeration of knots and links. In: Computational Problems in Abstract Algebra (Pergamon, 1970).

6. J.W. Alexander. Topological invariants of knots and links. Trans. Amer. Math. Soc. 30, 275 (1928).

7. V.F.R. Jones. A polynomial invariant for knots and links via von Neumann algebras Bull. AMS 12, 103 (1985).

8. P. Freyd, D. Yetter, J. Hoste, W.B.R. Lickorish, K. Millet, A. Ocneanu. A new polynomial invariant of knots and links. Bull. AMS 12, 239 (1985).

9. T.J. Rivlin. Chebyshev Polynomials: From Approximation Theory to Algebra and Number Theory (Wiley, 1990).

10. A.M. Gavrilik, A.M. Pavlyuk. On Chebyshev polynomials and torus knots. Ukr. J. Phys. 55, 129 (2010).

11. A.M. Gavrilik, A.M. Pavlyuk. Alexander polynomial invariants of torus knots $\mathrm{T}(\mathrm{n}, 3)$ and Chebyshev polynomials. Ukr. J. Phys. 56, 680 (2011).

12. A.M. Pavlyuk. On $T(n, 4)$ torus knots and Chebyshev polynomials. Ukr. J. Phys. 57, 439 (2012).

13. A.M. Pavlyuk. Polynomial invariants of torus knots and $(p, q)$-calculus. Algebras, Groups and Geometries 31, 175 (2014).

14. D. Rolfsen. Knots and Links (Publish or Perish, 1976).

15. W.B.R. Lickorish. An Introduction to Knot Theory (Springer, 1997).

Received 05.02.18

\section{А.М. Павлюк}

\section{УЗАГАЛЬНЕНІ ЕКВІДИСТАНТНІ ПОЛННОМИ} ЧЕБИШОВА І ВУЗЛОВІ ІНВАРІАНТИ АЛЕКСАНДЕРА

Р е $з$ ю м е

Вводяться узагальнені еквідистантні поліноми Чебишова $T^{(k, h)}$ роду $k$ із гіперроду $h$, де $k, h$ - додатні цілі числа. Вони отримані шляхом узагальнення стандартних поліномів Чебишова першого і другого родів та монічних поліномів Чебишова першого і другого родів. Це узагальнення виконується в двох напрямках. Горизонтальне узагальнення здійснюється шляхом введення поняття гіперроду $h$, і розширення множини його значень до нескінченності. Вертикальне узагальнення передбачає розширення множини значень роду $k$ до нескінченності методом еквідистантних коефіцієнтів. Досліджено деякі зв'язки введених поліномів 3 вузловими інваріантами Александера.

ISSN 2071-0194. Ukr. J. Phys. 2018. Vol. 63, No. 6 